\title{
Prognostic Scoring System Development for Malignant Spinal Cord Compression
}

\author{
Chaichana Chantharakhit*, Nantapa Sujaritvanichpong
}

\begin{abstract}
Objective: Although many prognostic scoring systems have been used to predict survival of malignant spinal cord compression (MSCC) patients, some previous data have shown that the accuracy of the scoring system remains problematic. Current advanced cancer therapies may influence the altered survival predictions. The aim of this study was to develop a new prognostic scoring system for higher accuracy of survival prediction in patients with malignant spinal cord compression (MSCC). Methods: Data were collected from 89 patients diagnosed with MSCC in 20182020. Potential clinical factors were analyzed using univariate and multivariate Cox's regression analysis. The selected logistic coefficients were transformed into a prognostic predictive scoring system. Internal validation was performed using the bootstrapping procedure. Results: According to multivariate Cox's regression analysis, 9 potential prognostic factors were obtained, i.e. Neutrophil-to-Lymphocyte ratio $>3.6$, breast cancer, lung cancer, other types of cancer (except prostate cancer), male, complete paralysis, spinal metastases in three levels, hypercalcemia, and no further systemic treatment. The data was developed into a Buddhasothorn Hospital Malignant Spinal Cord Compression (BSH-MSCC) score with an interval of $0-52$ points $(\mathrm{AUC}=0.77$; AUC to predict short-term survival $=0.93)$. When using the cut-off point $>18$ to predict short-term survival, $\mathrm{AUC}=0.84$, sensitivity $=81.5 \%$, specificity $=85.7 \%, \mathrm{PPV}=89.8 \%$, and NPV $=75.0 \%$. Internal validation with 1,000 bootstrap resampling showed good discrimination. Conclusion: BSH-MSCC score had a simplified score and high accuracy. The new tool is more accurate and can help decision-making for better treatment using a multidisciplinary approach.
\end{abstract}

Keywords: Prognostic score- malignant spinal cord compression- treatment modality

Asian Pac J Cancer Prev, 23 (2), 623-630

\section{Introduction}

Malignant spinal cord compression (MSCC) is an emergency in patients with cancer. The disease is usually caused by extradural metastases because delayed diagnosis and treatment can result in irreversible paralysis. For the symptoms of MSCC, patients usually have a typically back pain, depending on the location within the spine. Neurological deficits, such as weakness, tingling or numbness in the arms or legs may also develop. These neurological deficits tend to occur several weeks or months following the onset of back pain. Frequent locations of MSCC are the thoracic (60\%), lumbosacral $(30 \%)$, and cervical (10\%) spine, respectively (Cowap et al.,2000). MSCC is found in 5\% of all patients with cancer and, in $20 \%$ of them, can be an initial manifestation (Boussios et al., 2018).

Any patient suspected of spinal cord compression should undergo a magnetic resonance imaging (MRI) as soon as possible to determine the location and severity of the spinal cord lesion and to plan treatment. The treatment of MSCC depends mainly on the prognosis, neurological state, and recovery potential.

When MSCC is diagnosed, local management is required, i.e. palliative radiation, surgical posterior decompression with or without instrumentation, or total en bloc spondylectomy (Boussios et al., 2018). Decisionmaking on treatment approaches is up to prognostic prediction, e.g. palliative radiation only without surgery in case the survival rate is less than 6 months or in case of non-regeneration or select single-stage posterolateral transpedicular corpectomy and fusion in patients with pathological conditions around the thoracolumbar spine.

Therefore, there is a large amount of research on the prognostic prediction scoring system to help predict survival in patients with MSCC and aid decision-making for proper treatment planning, e.g. Tokuhashi et al., (1990), Bauer et al., (1995), Sioutos et al., (1995), Tomita et al., (2001), Katagiri et al., (2005), van der Linden et al., (2005), revised Tokuhashi et al., (2005), Oswestry Spinal Risk Index (Balain et al., 2013), Bollen et al., (2014), revised Katagiri et al., (2014), New England Spine Metastasis (NESMS) Score (Schoenfeld et al., 2016) and SORG machine-learning (ML) algorithms (Karhade 
et al., 2019). Even so, the scoring system with the most external validation is the Tokuhashi scoring system, which revised the prognostic model in 2005 for better accuracy of survival prediction (Owari et al., 2020). However, there was data for external validation of the Tokuhashi scoring system by Zoccali et al., (2016) and HernandezFernandez et al., (2012), who found that the Tokuhashi score was not accurate for validation. Although other scoring systems have been developed, such as the SORG nomogram, NESMS, modified Bauer, Katagiri, they were able to predict survival accurately but lacked neurological deficit, which is an important factor to be evaluated. In addition, the Katagiri score has the disadvantage that bone metastases are recorded as metastases to the entire skeleton, not just the spine.

Therefore, this study aimed to develop a new prognostic scoring system, called the Buddhasothorn Hospital Malignant Spinal Cord Compression score (BSHMSCC score), for higher accuracy of current survival prediction.

\section{Materials and Methods}

To develop a new prognostic scoring system, called the BSH-MSCC score, that helps to predict survival in patients with MSCC for surgical consideration. For the objective design, this is prognostic research, with the study base as a retrospective observational cohort study. We conducted this retrospective chart reviews study in compliance with the principles of the Declaration of Helsinki. The study's protocol was reviewed and approved by the Institutional Review Board number BSH-IRB 017/2564. The IRB has determined that formal consent is not required. The implementation process is described as follows.

1. Basic data of patients with MSCC were collected, i.e. sex, age, weight, height, type of cancer, site of metastasis, brain metastasis, ECOG performance status, date of death, Neutrophil-to-lymphocyte ratio (NLR), level of albumin, the number of metastatic spinal cords, neurological deficit, level of calcium, palliative radiation, and systemic cancer treatment after diagnosis of MSCC in accordance with the hospital-cancer database between January 2018 - December 2020.

2. Dates of death were verified and collected from the database of the verification room that connected with the database of civil registration in accordance with ID cards as recorded in the Bureau of Registration Administration (BORA), Department of Provincial Administration, Ministry of Interior, Thailand. Only causes of death from cancer were analyzed.

\section{Patients}

All data in the hospital-cancer database of patients diagnosed with MSCC between January 2018 and December 2020.

\section{Inclusion criteria}

1. Data of patients aged 18 years and over.

2. Patients with clinical history, i.e. basic data, diagnosis history, and potential prognostic predictors (sex, age, weight, height, type of cancer site of metastasis, brain metastasis, ECOG performance status, date of death, Neutrophil-to-lymphocyte ratio (NLR), level of albumin, the number of metastatic spinal cords, neurological deficit, level of calcium, palliative radiation, systemic cancer treatment after diagnosis of MSCC.

\section{Exclusion criteria}

1. Patients with incomplete/missing/lost data.

2. Patients who started treatment at other hospitals.

\section{Statistical analyses}

Step 1: Potential variables of clinical descriptions affecting survival in patients with MSCC were analyzed to find the relationship and directions affecting their survival by univariate Cox's proportional hazards regression analysis and multivariate Cox's proportional hazards regression analysis, that is, an exploratory model, to find potential prognostic factors. The significance level or alpha level is 0.05 .

Step 2: The factors related to multivariate Cox's proportional hazards regression model were transformed into a score by simplified risk score transformation. Each item was then assigned with specific score derived from the logistic regression coefficients of the multivariable model. The regression coefficient of each item was divided by the lowest coefficient and then rounded up to the nearest integer for developing the prognostic prediction scoring system, called the BSH-MSCC score.

Step 3: The value of the area under the ROC curve of the BSH-MSCC score was calculated to predict survival and find an appropriate cut-off point. Simultaneously, sensitivity, specificity, positive predictive value (PPV), negative predictive value (NPV), the likelihood ratio for a positive test $(\mathrm{LR}+)$, and likelihood ratio for a negative test (LR-) were also displayed.

Step 4: Internal validation was tested by calibration curve, along with statistical analysis by the HosmerLemeshow goodness-of-fit statistic test to estimate the accuracy of the developed prognostic prediction model (BSH-MSCC score).

Statistical analyses were performed using STATA version 16 (StataCorp, TX, USA).

\section{Results}

\section{Prognostic model development}

The data of 89 patients with MSCC were collected and classified by primary cancer, as in Table 1 . In previous studies, the Neutrophil-to-Lymphocyte ratio (NLR) was considered a biomarker of systemic inflammation and shown to predict survival in patients with metastatic cancer (Chantharakhit and Sujaritvanichpong, 2020; Templeton et al., 2014; Guthrie et al., 2013) . The cut-off points of NLR in those studies were uncertain. Some were over 3 points or even over 3.3 points. No matter what, it was found that high NLR is usually related to poor prognostic outcomes (Faria et al., 2016; Azab et al., 2012; Krenn-Pilko et al., 2014). This study used the cut-off point of NLR > 3.6, with sensitivity $81.69 \%$, specificity $38.89 \%$, likelihood ratio (LR+) 1.34, and likelihood ratio (LR-) 0.47.

Potential variables were analyzed to find the relationship 


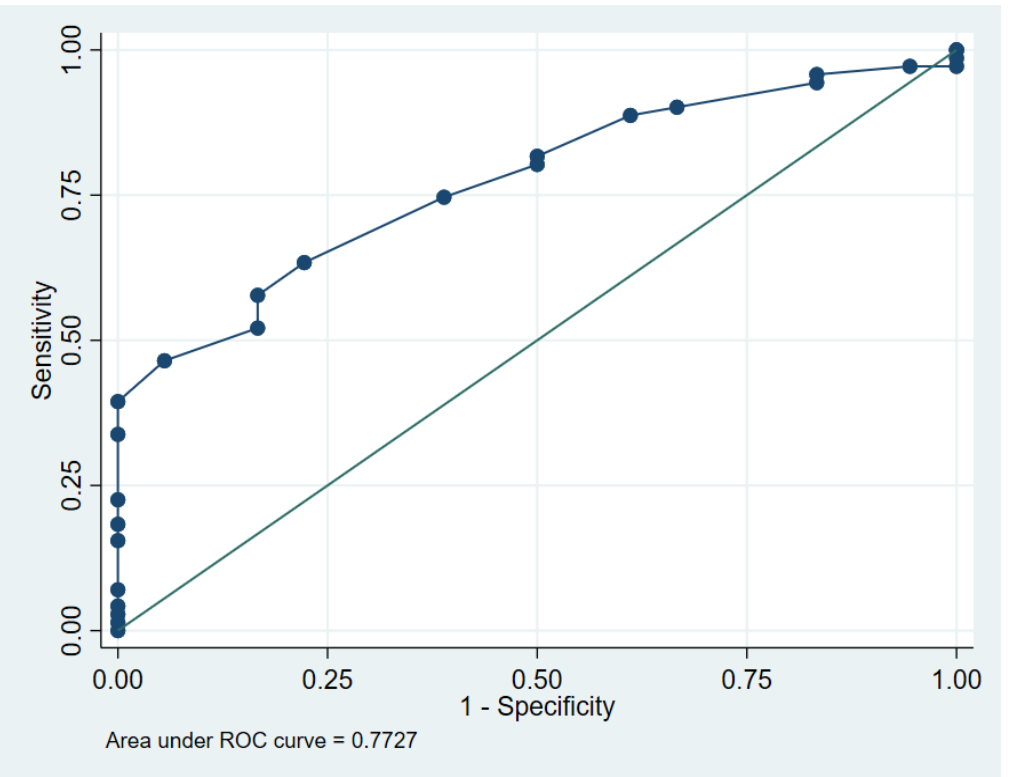

Figure 1. AUC of the BSH-MSCC Score for Predicted Survival $=0.77$

Table 1. Primary Cancer in Patients with Spinal Cord Metastasis $(\mathrm{N}=89)$

\begin{tabular}{lc}
\hline Primary cancer & Number $(\%)$ \\
\hline Lung cancer & $29(32.58)$ \\
Breast cancer & $15(16.85)$ \\
Prostate cancer & $8(8.99)$ \\
Other cancer & \\
$\quad$ Colorectal cancer & $8(8.99)$ \\
Upper gastrointestinal tract cancer & $6(6.74)$ \\
Hepatocellular carcinoma & $4(4.49)$ \\
Unknown primary cancer & $7(7.86)$ \\
Hematologic malignancy & $4(4.49)$ \\
Head and neck cancer & $4(4.49)$ \\
Renal cell carcinoma & $2(2.25)$ \\
Gynecologic malignancy & $2(2.25)$ \\
\hline
\end{tabular}

with survival in patients with MSCC by univariate and multivariate Cox's proportional hazards regression analysis. Potential prognostic factors from univariate Cox's proportional hazards regression analysis were NLR $>3.6$, prostate cancer, hypercalcemia $(>10.5 \mathrm{mg} / \mathrm{dL})$, no further systemic treatment, receipt of radiotherapy. Potential prognostic factors from multivariate Cox's proportional hazards regression analysis were found, i.e. breast cancer, lung cancer, other cancers (except prostate cancer), male, complete paralysis, hypercalcemia $>10.5$ $\mathrm{mg} / \mathrm{dL}$, and no further systemic, as shown in Table 2.

\section{Score transformation (BSH-MSCC score)}

Each potential predictor in the multivariable model was assigned with a specific score derived from the multivariate Cox's proportional hazards regression coefficient (Table 3). The regression coefficient of each item was divided by

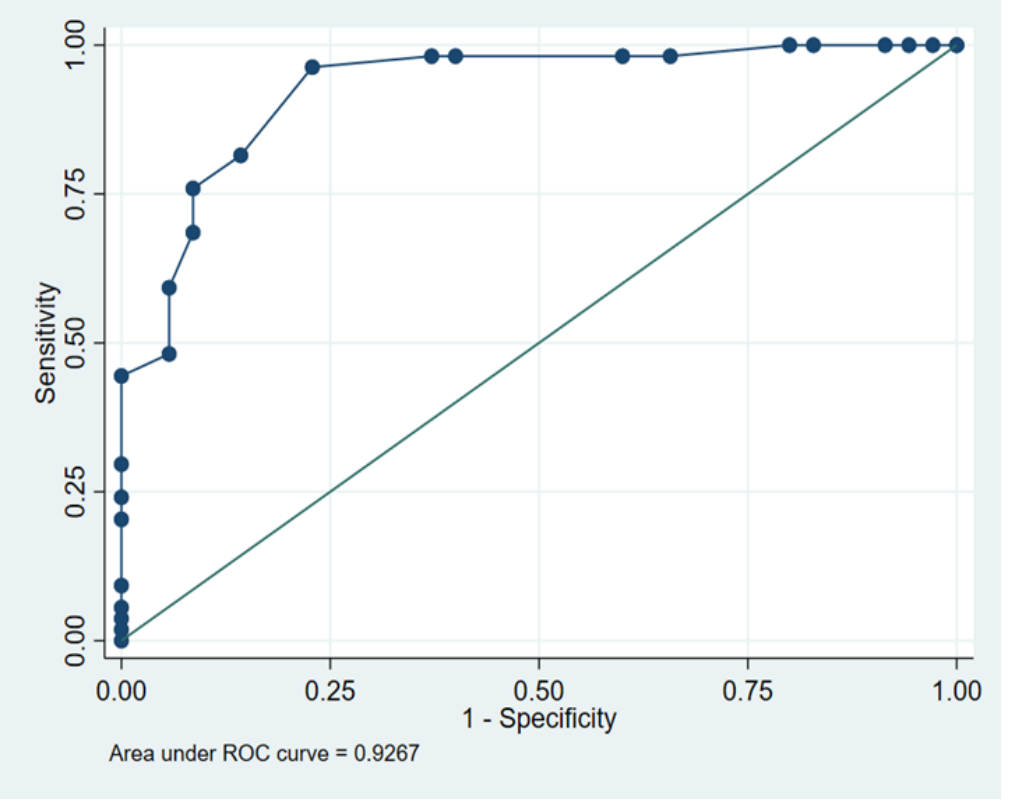

Figure 2. AUC of the BSH-MSCC Score for Predicted Survival Less than 6 Month $=0.93$ 
Table 2. Univariate and Multivariate Cox's Proportional Hazards Regression Analysis of MSCC and Variable Factors.

\begin{tabular}{|c|c|c|c|c|c|c|}
\hline Variables & $\begin{array}{l}\text { Crude hazard } \\
\text { ratio }\end{array}$ & $\begin{array}{l}\text { 95\% Confidence } \\
\text { Interval }\end{array}$ & p-value & $\begin{array}{c}\text { Adjusted } \\
\text { hazard ratio }\end{array}$ & $\begin{array}{l}\text { 95\% Confidence } \\
\text { Interval }\end{array}$ & p-value \\
\hline NLR $<3.6$ & 1 & reference & - & 1 & reference & - \\
\hline NLR $>3.6$ & 1.89 & $1.01-3.54$ & $0.045^{*}$ & 1.33 & $0.66-2.68$ & 0.421 \\
\hline Prostate cancer & 0.32 & $0.11-0.91$ & $0.032 *$ & Omitted & & \\
\hline Breast cancer & 0.63 & $0.31-1.27$ & 0.194 & 4.79 & $1.25-18.39$ & $0.022 *$ \\
\hline Lung cancer & 0.94 & $0.56-1.60$ & 0.833 & 7.34 & $2.23-24.13$ & $0.001 *$ \\
\hline Other cancer & 1 & reference & - & 3.83 & $1.22-12.00$ & $0.021 *$ \\
\hline Female & 1 & reference & - & 1 & reference & - \\
\hline Male & 0.92 & $0.57-1.47$ & 0.716 & 1.94 & $1.07-3.52$ & $0.029 *$ \\
\hline $\mathrm{ECOG}<2$ & 1 & reference & - & 1 & reference & - \\
\hline $\mathrm{ECOG}>2$ & 1.27 & $0.79-2.06$ & 0.322 & 0.6 & $0.33-1.07$ & 0.086 \\
\hline Age $<60$ & 1 & reference & - & 1 & reference & - \\
\hline Elderly (>60 year) & 1.15 & $0.71-1.86$ & 0.568 & 1.11 & $0.65-1.89$ & 0.698 \\
\hline Incomplete paralysis & 1 & reference & - & 1 & reference & - \\
\hline Complete paralysis & 1.6 & $1.00-2.57$ & 0.05 & 2.07 & $1.16-3.70$ & $0.014^{*}$ \\
\hline $0-2$ level spine metastasis & 1 & reference & - & 1 & reference & - \\
\hline Triple level spine metastasis & 1.13 & $0.70-1.80$ & 0.62 & 1.53 & $0.90-2.60$ & 0.117 \\
\hline No hypercalcemia & 1 & reference & - & 1 & reference & - \\
\hline Hypercalcemia (>10.5 mg/dL) & 7.68 & $2.54-23.21$ & $<0.001^{*}$ & 4.31 & $1.33-13.92$ & $0.015^{*}$ \\
\hline Known case cancer & 1 & reference & - & 1 & reference & - \\
\hline $\begin{array}{l}\text { Clinical presentation with MSCC } \\
\text { before cancer diagnosis }\end{array}$ & 1.27 & $0.78-2.06$ & 0.333 & 0.9 & $0.51-1.59$ & 0.715 \\
\hline Further systemic treatment & 1 & reference & - & 1 & reference & - \\
\hline No further systemic treatment & 3.34 & $1.96-5.67$ & $<0.001^{*}$ & 7.43 & $3.55-15.56$ & $<0.001 *$ \\
\hline Not receiving radiotherapy & 1 & reference & - & & & \\
\hline Receiving radiotherapy & 0.43 & $0.26-0.70$ & $0.001 *$ & Not analysis & & \\
\hline
\end{tabular}

* Statistically significant p-values

the lowest coefficient and then rounded up to the nearest integer for developing the prognostic prediction scoring system. The scoring scheme had a total score ranging from zero to 52. For the discriminative ability, the area under the
ROC curve (AUC) for the score-based logistic regression model $=0.77$ (95\% CI 0.67-0.88) as shown in Figure 1, and AUC for the score-based predicted short-term survival less than 6 months $=0.93(95 \%$ CI 0.87-0.98) (Figure 2).

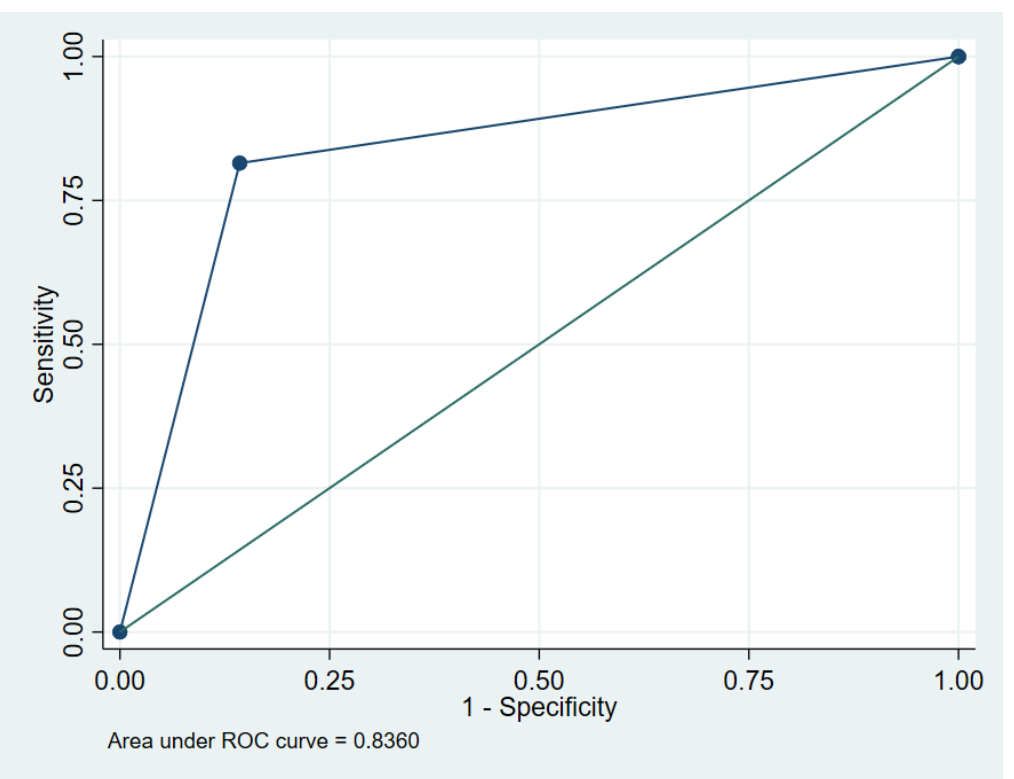

Figure 3. AUC of the BSH-MSCC Score at the Cut-Off Point $>18$ for Predicted Short Survival $<6$ month $=0.84$, sensitivity $81.5 \%$, specificity $85.7 \%$, positive predictive value (PPV) $89.8 \%$, and negative predictive value (NPV) $75.0 \%$ 
Table 3. Risk Score Derivation Using Multivariate Logistic Regression Coefficients (BSH-MSCC Score)

\begin{tabular}{lcccc}
\hline Potential Predictors & Coefficients & $95 \%$ Confidence Interval & p-value & Score \\
\hline NLR $>3.6$ & 0.20 & -1.36 & 0.566 & 1 \\
Breast cancer & 1.67 & $0.33-3.00$ & 0.014 & 8 \\
Lung cancer & 1.89 & $0.71-3.07$ & 0.002 & 10 \\
Other cancer (except prostate cancer) & 1.32 & $0.19-2.45$ & 0.022 & 7 \\
Male & 0.74 & $0.16-1.31$ & 0.011 & 4 \\
Complete paralysis & 0.52 & -1.08 & 0.061 & 3 \\
Triple level spine metastasis & 0.44 & -1.02 & 0.088 & 2 \\
Hypercalcemia (>10.5 mg/dL) & 1.58 & $0.44-2.70$ & 0.006 & 8 \\
No further systemic treatment & 1.80 & $1.13-2.47$ & $<0.001$ & 9 \\
\hline
\end{tabular}

According to the distribution of BSH-MSCC, it was a normal distribution, with the point where the score was over 18 , the cut-off point with sensitivity $81.48 \%$, and specificity $85.71 \%$ (Table 4 ). When analyzing the efficiency of BSH-MSCC at the cut-off point to predict survival less than 6 months, it was found that $\mathrm{AUC}=0.84$, sensitivity $81.5 \%$, specificity $85.7 \%$, positive predictive value (PPV) $89.8 \%$, negative predictive value (NPV) $75.0 \%$, likelihood ratio (LR+) 5.70, likelihood ratio (LR-) 0.22 (Figure 3). The measurement of the calibration is illustrated with the risk curve plot as shown in Figure 4.

Table 4. Detailed Report of Sensitivity and Specificity for Each cut-off Point

\begin{tabular}{|c|c|c|c|c|}
\hline Cutpoint & Sensitivity & Specificity & LR+ & LR- \\
\hline$>=5$ & 100 & 0 & 1 & \\
\hline$>=6$ & 100 & 2.86 & 1.0294 & 0 \\
\hline$>=7$ & 100 & 5.71 & 1.0606 & 0 \\
\hline$>=8$ & 100 & 8.57 & 1.0937 & 0 \\
\hline$>=10$ & 100 & 17.14 & 1.2069 & 0 \\
\hline$>=11$ & 100 & 20 & 1.25 & 0 \\
\hline$>=13$ & 98.15 & 34.29 & 1.4936 & 0.054 \\
\hline$>=14$ & 98.15 & 40 & 1.6358 & 0.0463 \\
\hline$>=15$ & 98.15 & 60 & 2.4537 & 0.0309 \\
\hline$>=16$ & 98.15 & 62.86 & 2.6424 & 0.0295 \\
\hline$>=17$ & 96.3 & 77.14 & 4.213 & 0.048 \\
\hline$>=18$ & 81.48 & 85.71 & 5.7037 & 0.216 \\
\hline$>=19$ & 75.93 & 91.43 & 8.858 & 0.2633 \\
\hline$>=20$ & 68.52 & 91.43 & 7.9938 & 0.3443 \\
\hline$>=21$ & 59.26 & 94.29 & 10.3704 & 0.4321 \\
\hline$>=22$ & 48.15 & 94.29 & 8.4259 & 0.5499 \\
\hline$>=23$ & 44.44 & 100 & & 0.5556 \\
\hline$>=24$ & 29.63 & 100 & & 0.7037 \\
\hline$>=25$ & 24.07 & 100 & & 0.7593 \\
\hline$>=26$ & 20.37 & 100 & & 0.7963 \\
\hline$>=27$ & 9.26 & 100 & & 0.9074 \\
\hline$>=30$ & 5.56 & 100 & & 0.9444 \\
\hline$>=31$ & 3.7 & 100 & & 0.963 \\
\hline$>=32$ & 1.85 & 100 & & 0.9815 \\
\hline$>=32$ & 0 & 100 & & 1 \\
\hline
\end{tabular}

\section{Internal validation}

The median Hosmer-Lemeshow test p-value was 0.96, indicating good calibration. Internal validation of the derived prognostic model was performed via a bootstrap resampling procedure with 1,000 replicates. The apparent $\mathrm{C}$-statistics and test $\mathrm{C}$ statistics were 0.77 (95\% CI 0.66$0.88, \min 0.54, \max 0.95$ ) and 0.77 (95\% CI 0.71-0.83, min 0.60 , max 0.81 ), respectively. The $\mathrm{C}$-statistic optimism was 0.03 (min -0.18 , max 0.23 ).

\section{Discussion}

The researchers developed the BSH-MSCC score, a new prognostic scoring system, from potential factors obtained by multivariate analysis. The factors of biomarkers related to prognosis, i.e. serum calcium level and NLR, were also brought for analysis. It was found that the BSH-MSCC score at a cut-off point over 18 was the cut-off value related to poor prognosis of short-term survival less than 6 months (AUC for predicted survival $=0.77$, and $\mathrm{AUC}$ of the scoring system for predict short survival $=0.93$ ). When using the cut-off point over 18 for predicted survival less than 6 months, it was found that AUC $=0.84$, sensitivity $81.5 \%$, specificity $85.7 \%$, positive predictive value (PPV) $89.8 \%$, and negative predictive value (NPV) $75.0 \%$.

The Tokuhashi score is the first prognostic score developed in 1990 to predict survival in MSCC. It was revised in 2005. It is the scoring system with the most external validation. Data from some studies found accuracy, while others found low accuracy (Lee et al., 2015). The parameters that the Tokuhashi score used for analysis still lacks the factors of a biomarker to blood chemistry related to prognostic factors in patients with cancer, e.g. Neutrophil-to-Lymphocyte ratio (NLR), Platelet-to-Lymphocyte ratio (PLR) (Wang et al., 2018), malignancy-associated hypercalcemia (Ramos et al., 2017), prognostic nutritional index (PNI) (Sun et al., 2017), advanced lung cancer inflammation index (ALI) (Chantharakhit and Sujaritvanichpong, 2021). When analyzing the efficiency of the prognostic scoring system at the cut-off point to predict survival less than 6 months by meta-analysis, low accuracy was found (Lee et al., 2015). This might be due to the current chance of survival in patients with cancer compared to the past. Therefore, the cut-off point could be different from 


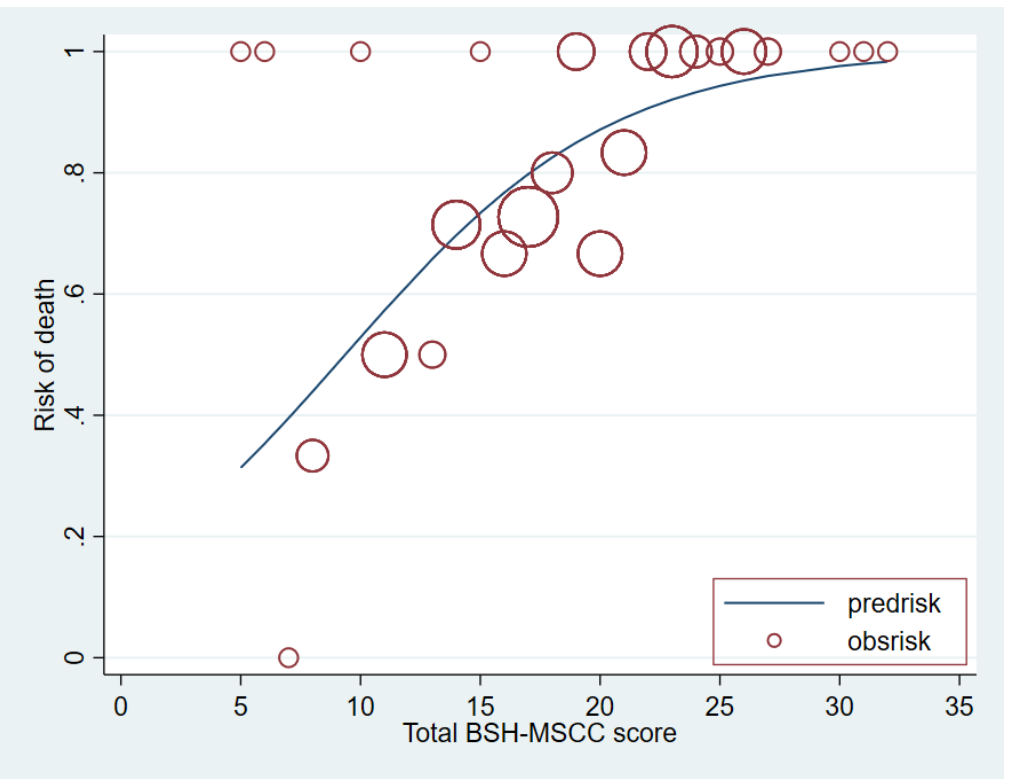

Figure 4. The Risk Curve Displaying Observed Risk (Circle) Versus the Score for Predicted Risk (Solid Line) of Death. The size of the circle represents the frequency of death in each score.

previously for predicting current short survival because of more advanced treatment, particularly systemic cancer treatment. In this regard, the Tokuhashi scoring system still lacks the factors of systemic cancer treatment to analyze survival prediction in patients. Therefore, the development of the new prognostic scoring system by the BSH-MSCC score also relied on the factors of biomarker, i.e. NLR, hypercalcemia, and the factors of systemic cancer treatment to analyze survival outcomes. This is a benefit of the BSH-MSCC scoring system. Although other scoring systems have been developed, such as the SORG nomogram, NESMS, modified Bauer, Katagiri, they were able to predict survival accurately but lacked neurological deficit, which is an important factor to be evaluated. In addition, the Katagiri score has the disadvantage that bone metastases are recorded as metastases to the entire skeleton, not just the spine.

Treatment of MSCC by radiotherapy is already a standard treatment in MSCC and an acceptable factor with the outcome as a protective factor for survival in patients with MSCC (Rades et al., 2010; Rades et al., 2011). Therefore, this factor was not used as a potential variable for multivariate analysis. Also, radiotherapy was not used as a factor for preoperative assessment of prognosis for the prognostic scoring system.

Despite the current concept that a scoring system for a specific type of cancer should be accurate/precise, scoring systems for a combination of several types of cancer are less useful due to the different nature and treatment for each type of cancer (Owari et al., 2018). The advantage of a cancer-specific prognostic scoring system is its accuracy to predict each type of cancer. In terms of disadvantages, a large number of scoring systems must be developed in accordance with the numerous types of cancer, resulting in difficulty. The BSH-MSCC scoring system is a nonspecific cancer scoring system. One advantage is that it can be used for all types of cancer and can be used simply in clinical practice. The researchers tried to adjust the effects of the factors differently influencing survival up to types of cancer and systemic treatment and brought them for analysis as part of the parameters in the scoring system. It can be seen that prostate cancer was not used as a factor to predict survival due to its slow progression from other types of cancer until it became a protective factor for survival by univariate and multivariate Cox's proportional hazards regression analysis with the parameters of systemic cancer treatment in patients, and with biomarker influencing prognosis.

This study had several limitations. First, it was a retrospective study conducted at a single institution and included patients with MSCC from several types of cancer. The second limitation was that treatment for MSCC was not consistent at our hospital. The number of patients receiving surgery was very small $(4.5 \%)$, while those receiving radiation amounted to $43.8 \%$. Therefore, the groups of patients in this research were not balanced in terms of treatment approaches to be an optimal treatment strategy for MSCC. The third limitation was that this study involved a non-specific prognostic scoring system for cancer with both advantages and limitations. The fourth limitation of the study is the small sample size. Therefore, further external validation is required with a larger number of patients, including those receiving surgical treatment, in order to confirm the accuracy of our scoring system.

The treatment aims for MSCC are to restore or preserve physical and neurological function, restoration or maintenance of spinal stability, pain control, and improvement of quality of life. The key principles of management for MSCC include an optimal level of surgical intervention in proportion to prognosis. Therefore, the development of an appropriate prognostic scoring system to predict survival is necessary.

In conclusion, the BSH-MSCC score may be useful for predicting life expectancy in patients with MSCC. However, using only a prognostic score for decision making may be insufficient. Decisions should also be 
made on an individual basis using a multidisciplinary approach and external validation is required.

\section{Author Contribution Statement}

Chaichana Chantharakhit: designed the study, reviewed the paper, collected data, analyzed data, draft manuscript preparation, edited the final version. Nantapa Sujaritvanichpong: collected data, reviewed the paper. All authors read an approved the final version.

\section{Acknowledgements}

The study protocol was approved by the Institutional Review Board of Buddhasothorn Hospital number BSHIRB 017/2564. This research received no specific grant from any funding agency in the public, commercial, or not-for-profit sectors.

\section{Conflict of interest}

The authors confirm that there are no relevant financial or non-financial competing interests to report and no conflicts of interest to declare.

\section{References}

Azab B, Bhatt VR, Phookan J, et al (2012). Usefulness of the neutrophil-to-lymphocyte ratio in predicting short- and long-term mortality in breast cancer patients. Ann Surg Oncol, 19, 217-24.

Balain B, Jaiswal A, Trivedi JM, et al (2013). The Oswestry Risk Index: an aid in the treatment of metastatic disease of the spine. Bone Joint J, 95-B, 210-6.

Bauer HC, Wedin R (1995). Survival after surgery for spinal and extremity metastases. Prognostication in 241 patients. Acta Orthop Scand, 66, 143-6.

Bollen L, van der Linden YM, Pondaag W, et al (2014). Prognostic factors associated with survival in patients with symptomatic spinal bone metastases: a retrospective cohort study of 1,043 patients. Neuro Oncol, 16, 991-8.

Boussios S, Cooke D, Hayward C, et al (2018). Metastatic spinal cord compression: Unraveling the Diagnostic and Therapeutic Challenges. Anticancer Res, 38, 4987-97.

Chantharakhit C, Sujaritvanichpong N (2020). Pretreatment absolute neutrophil-to-lymphocyte ratio (NLR) predict the risk for febrile neutropenia in the first cycle adjuvant chemotherapy for breast cancer. $A P J C B, 5,81-7$.

Chantharakhit C, Sujaritvanichpong N (2021). Prognostic impact of the advanced lung cancer inflammation index (ALI) in metastatic non-small cell lung cancer treated with first line chemotherapy. Asian Pac J Cancer Prev, 22, 1149-56.

Cowap J, Hardy JR, A'Hern R (2000). Outcome of malignant spinal cord compression at a cancer center: implications for palliative care services. J Pain Symptom Manage, 19, 257-64.

Faria SS, Fernandes PC Jr, Silva MJ, et al (2016). The neutrophil-to-lymphocyte ratio: a narrative review. Ecancermedicalscience, 10, 702.

Guthrie GJ, Charles KA, Roxburgh CS, et al (2013). The systemic inflammation-based neutrophil-lymphocyte ratio: experience in patients with cancer. Crit Rev Oncol Hematol, 88, 218-30.

Hernandez-Fernandez A, Vélez R, Lersundi-Artamendi A, Pellisé F (2012). External validity of the Tokuhashi score in patients with vertebral metastasis. J Cancer Res Clin Oncol,
138, 1493-500.

Karhade AV, Thio QCBS, Ogink PT, et al (2019). Predicting 90-day and 1-year mortality in spinal metastatic disease: Development and Internal Validation. Neurosurgery, 85, E671- 81.

Katagiri H, Okada R, Takagi T, et al (2014). New prognostic factors and scoring system for patients with skeletal metastasis. Cancer Med, 3, 1359-67.

Katagiri H, Takahashi M, Wakai K, et al (2005). Prognostic factors and a scoring system for patients with skeletal metastasis. J Bone Joint Surg Br, 87, 698-703.

Krenn-Pilko S, Langsenlehner U, Thurner EM, et al (2014). The elevated preoperative platelet-to-lymphocyte ratio predicts poor prognosis in breast cancer patients. Br J Cancer, 110, 2524-30.

Lee CH, Chung CK, Jahng TA, et al (2015). Which one is a valuable surrogate for predicting survival between Tomita and Tokuhashi scores in patients with spinal metastases? A meta-analysis for diagnostic test accuracy and individual participant data analysis. J Neurooncol, 123, 267-75.

Owari T, Miyake M, Nakai Y, et al (2018). A genitourinary cancer-specific scoring system for the prediction of survival in patients with bone metastasis: A Retrospective Analysis of Prostate Cancer, Renal Cell Carcinoma, and Urothelial Carcinoma. Anticancer Res, 38, 3097-103.

Owari T, Miyake M, Nakai Y, et al (2020). External validation of a genitourinary cancer-specific prognostic scoring system to predict survival for patients with bone metastasis (modified B-FOM scoring model): Comparison with other scoring models in terms of accuracy. J Bone Oncol, 26, 100344.

Rades D, Huttenlocher S, Bajrovic A, et al (2011). Surgery followed by radiotherapy versus radiotherapy alone for metastatic spinal cord compression from unfavorable tumors. Int J Radiat Oncol Biol Phys, 81, e861-8.

Rades D, Huttenlocher S, Dunst J, et al (2010). Matched pair analysis comparing surgery followed by radiotherapy and radiotherapy alone for metastatic spinal cord compression. J Clin Oncol, 28, 3597-604.

Ramos REO, Perez Mak M, Alves MFS, et al (2017). Malignancy-related hypercalcemia in advanced solid tumors: Survival Outcomes. J Glob Oncol, 3, 728-33.

Schoenfeld AJ, Le HV, Marjoua Y, et al (2016). Assessing the utility of a clinical prediction score regarding 30-day morbidity and mortality following metastatic spinal surgery: the New England Spinal Metastasis Score (NESMS). Spine J, 16, 482-90.

Sioutos PJ, Arbit E, Meshulam CF, Galicich JH (1995). Spinal metastases from solid tumors. Analysis of factors affecting survival. Cancer, 76, 1453-9.

Sun J, Wang D, Mei Y, et al (2017). Value of the prognostic nutritional index in advanced gastric cancer treated with preoperative chemotherapy. J Surg Res, 209, 37-44.

Templeton AJ, McNamara MG, Šeruga B, et al (2014). Prognostic role of neutrophil-to-lymphocyte ratio in solid tumors: a systematic review and meta-analysis. $J$ Natl Cancer Inst, 106, dju124.

Tokuhashi Y, Matsuzaki H, Oda H, Oshima M, Ryu J (2005). A revised scoring system for preoperative evaluation of metastatic spine tumor prognosis. Spine (Phila Pa 1976), 30, 2186-91.

Tokuhashi Y, Matsuzaki H, Toriyama S, Kawano H, Ohsaka S (1990). Scoring system for the preoperative evaluation of metastatic spine tumor prognosis. Spine (Phila Pa 1976), 15, 1110-3.

Tomita K, Kawahara N, Kobayashi T, et al (2001). Surgical strategy for spinal metastases. Spine (Phila Pa 1976), 26, 298-306. 
Chaichana Chantharakhit and Nantapa Sujaritvanichpong

van der Linden YM, Dijkstra SP, Vonk EJ, Marijnen CA, Leer JW (2005); Dutch Bone Metastasis Study Group. Prediction of survival in patients with metastases in the spinal column: results based on a randomized trial of radiotherapy. Cancer, 103, 320-8.

Wang J, Zhou X, He Y, et al (2018). Prognostic role of platelet to lymphocyte ratio in prostate cancer: A meta-analysis. Medicine (Baltimore), 97, e12504.

Zoccali C, Skoch J, Walter CM, et al (2016). The Tokuhashi score: effectiveness and pitfalls. Eur Spine J, 25, 673-8.

\section{c) (7) (8)}

This work is licensed under a Creative Commons AttributionNon Commercial 4.0 International License. 